\title{
NOVAS PROPOSTAS DE AVALIAÇÃO DA RESISTÊNCIA ESPECIAL DO JUDOCA
}

Rodrigo Ribeiro Rosa

Paulo Roberto de Oliveira

\section{Resumo}

As necessidades de se estabelecerem métodos confiáveis de controle do treinamento do atleta é uma das principais preocupações dos pesquisadores em Ciências do Desporto. Sendo assim, o objetivo desta pesquisa é formular novos processos de avaliação física, de modo a responder às especificidades do judô. O desempenho de dois grupos de judocas, diferenciados pelo nível competitivo, foi mensurado através de três testes específicos (Special Judo Fitness Test - SJFT, TC3 e TC15). Os resultados mostraram que: houve diferença significante entre os grupos no desempenho do TC3 $(\mathrm{p}<0,001)$ e TC15 ( $\mathrm{p}<0,05)$, mas não houve o mesmo no SJFT; TC3 apresentou alta fidedignidade. Através desses resultados, concluiu-se que TC3 é aplicável como nova proposta de avaliação do desempenho de atletas de judô.

\section{Palavras-Chave}

Judô; Avaliação; Lactato; Treinamento desportivo.

\section{NEW PURPOSES OF EVALUATION OF JUDOKA'S SPECIAL ENDURANCE}

Rodrigo Ribeiro Rosa

Paulo Roberto de Oliveira

\begin{abstract}
The need to establish a reliable method of controlling the athlete training is one of the main concerns of science sports researches. As a consequence, the purpose of this research is to formulate a new physical evaluation process, to answer the specificity of judo. The performance of 2 judoka's groups, with different competitive performance, was mentioned by 3 specific situations (Special Judo Fitness Test - SJFT, TC3 e TC15). The results showed that: there was a significant difference among groups in TC3 $(\mathrm{p}<0,001)$ and TC15 $(\mathrm{p}<0,05)$ performance, but there wasn't the same in SJFT; TC3 had a high reliability. According to this, we can conclude that TC3 is applicable as a new purpose of evaluation of judoka's performance.
\end{abstract}

\section{Key-Words}

Judo; Evaluation; Lactate; Sports training. 


\section{INTRODUÇÃO}

No atual cenário desportivo, as crescentes preocupações acerca da racionalização dos meios e métodos de preparação dos atletas, levaram os pesquisadores da ciência do treinamento desportivo a procurar regras gerais e especiais do processo de adaptação a longo prazo dos atletas às condições da atividade competitiva e à dinâmica da condição do atleta, em função da carga de treinamento proposta. No caso do judô, observa-se uma evolução significativa dos resultados competitivos de países como Rússia, Geórgia, França e Cuba, comparada ao Japão, que detém a hegemonia dentro do judô mundial, possivelmente, em virtude da crescente preocupação dos técnicos dos países citados em buscar soluções teóricas e práticas para a construção da programação e controle do treinamento, que possa suprir as necessidades do desporto de alto nível.

Dentro da literatura específica sobre avaliação no judô, é possível observar a seguinte particularidade: ao longo da história, os trabalhos se detinham em observações do desempenho em testes gerais, tanto na capacidade aeróbia (DEMEERSMAN; RUHLING, 1977; TAYLOR; BRASSARD, 1981; TUMILTY et al., 1986; SIKORSKI et al., 1987; THOMAS et al., 1989; LITTLE, 1991; CALLISTER et al., 1991; BORKOWSKI et al., 2001), como na anaeróbia (BAR-OR, 1987). Nesse panorama, destaca-se o único método existente validado para o controle de treinamento em judô, oriundo dos estudos conduzidos por Sterkowicz (1995) apud Franchini (2001). Porém, considerando-se os referenciais teóricos, existem certos pontos que fogem à especificidade do desporto, como deslocamentos em forma de corrida, não condizentes com o observado na modalidade. Tais aspectos são tratados com grande ênfase pelos cientistas do desporto, como Kuznetsov (1986), Verkhoshansky (1990), Matveev (1980), Bompa (1999) e Zatsiorsky (1999), procurando levar o Princípio da Especificidade como um dos pontos fundamentais na estruturação de elementos que possam ser válidos na avaliação e prescrição do treinamento de atletas de alto nível.

\section{OBJETIVOS}

O presente estudo teve como objetivo apresentar novos protocolos de avaliação de rendimento do atleta, levando-se em consideração fatores como estrutura temporal observada em combates e utilização de alavancas específicas empregadas na luta. 


\section{METODOLOGIA}

Sujeitos da pesquisa

O grupo de estudo da pesquisa foi composto por 11 atletas do sexo masculino, todos com no mínimo, graduação de faixa marrom $\left(1^{\circ} \mathrm{kyu}\right)$ e 18 anos de idade completos. Os atletas foram subdivididos em dois grupos: seis atletas no grupo um (G1) e cinco atletas no grupo dois (G2). Além disso, um questionário foi aplicado, contendo perguntas sobre a vida desportiva do atleta, com respeito ao tempo de prática do judô, graduação atual no judô, freqüência atual de treinamento, títulos conquistados e número de desportos praticados regularmente durante a vida.

O trabalho foi devidamente aprovado pelo Comitê de Ética em Pesquisa (processo $n^{\circ} 177 / 2005$ ) envolvendo seres humanos e todos os atletas participantes foram devidamente informados e esclarecidos quanto à realização dos testes, assinando Termo de Consentimento Livre e Esclarecido para a realização do trabalho.

\section{SPECIAL JUDO FITNESS TEST (SJFT)}

O SJFT segue o modelo proposto por Sterkowicz (1995) apud Franchini (2001). Para aferição da FC foi utilizado monitor específico da marca Polar, modelo Accurex (Electro Oy, Finlândia). O número de golpes aplicados em tempo definido (três séries de esforço máximo com duração de 15, 30 e 30 segundos, respectivamente, intercalados com 10 segundos de pausa, contabilizando 95 segundos), e a freqüência cardíaca (FC) pós-esforço foram utilizados como variáveis para o cálculo do índice que determina o nível de rendimento do atleta.

\section{TC3 e TC15}

O desenvolvimento do TC3 e TC15 tem a seguinte estrutura:

1 - o posicionamento de três judocas que serão arremessados (chamados de $u k e ̂$ ), em disposição triangular, com 3 (três) metros de distância entre si. No centro do triângulo, encontra-se o atleta ser avaliado (ou tori), sendo que todos os envolvidos na situação apresentada são de massa corporal semelhante.

2 - Com relação ao âmbito técnico optou-se pelo golpe ippon seoi nague, que tem uma das maiores incidências de aplicação, conforme atestam Sterkowicz e Franchini (2000) e Silva (2002), em observações das lutas em atletas de nível olímpico e mundial. 
3 - O teste propõe a aplicação de séries de esforço, na máxima intensidade possível, divididos em duas situações: 3 séries (TC3) e 15 séries (TC15) de 20 segundos de esforço. Em cada série, é registrado o número de arremessos realizados; entre cada série, ocorre intervalo de recuperação passiva de 10 segundos. Para a análise do desempenho no TC3 e no TC15, leva-se em consideração o volume total de arremessos realizados.

O esquema do modelo do TC3 e TC15 é descrito na Figura 1. As características do teste propõem-se em mostrar o desempenho em função do nível de esforço empregado pelo judoca, tanto na rapidez de execução dos golpes, quanto na capacidade de manutenção da intensidade do esforço durante o teste.

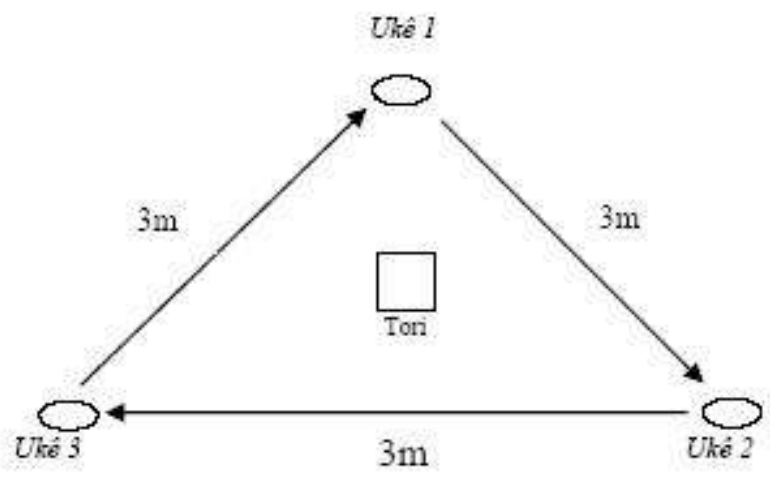

FIGURA 1 - Desenho esquemático do TC3 e TC15

\section{ANÁLISE ESTATÍSTICA}

De posse dos dados, realizou-se o teste de normalidade; no caso, utilizou-se o teste de Shapiro-Wilk. Levando em consideração os resultados encontrados, os testes não-paramétricos foram adotados para testar as diferenças entre os grupos quanto às características morfológicas e marcadores externos (desempenho nos testes).

Para testar diferenças entre os grupos, foi utilizado o teste de Mann-Whitney, com nível de significância $\mathrm{p}<0,05$.

Para testar a fidedignidade do teste TC3, utilizou o coeficiente de correlação intraclasse (CCI) entre os resultados do TC3 e as três primeiras séries do TC15, sendo o grupo de judocas tratado como grupo único. 


\section{RESULTADOS}

Caracterização do grupo de trabalho

Os dados a respeito das características morfológicas dos atletas estão dispostos na tabela 1, com os valores sobre idade (em anos), massa corporal (MC, em quilogramas) e altura (ALT, em metros).

TABELA 1 - Caracteristicas morfologicas dos judocas

\begin{tabular}{ccccc}
\hline Grupos & Valores & Idade & MC $(\mathrm{kg})$ & ALT $(\mathrm{m})$ \\
\hline \multirow{3}{*}{ G1 } & MÉDIA & 21,65 & 75,25 & 1,76 \\
& DP & 3,54 & 8,13 & 0,06 \\
& MEDIANA & 21,83 & 77,15 & 1,76 \\
\hline \multirow{2}{*}{ G2 } & MÉDIA & 28,28 & 79,70 & 1,72 \\
& DP & 5,28 & 11,13 & 0,02 \\
& MEDIANA & 30,00 & 83,50 & 1,72 \\
\hline
\end{tabular}

$+p<0,05$ entre GI $G_{2}$

$\mathrm{Na}$ Tabela 2 e 3 estão registradas as respostas do questionário a respeito da vida desportiva dos atletas do G1 e G2, respectivamente. Dos 11 atletas, apenas um não respondeu ao questionário.

TABELA 2 - Respostas sobre a vida desportiva dos atletas do G1

\begin{tabular}{|c|c|c|c|c|c|c|c|c|c|c|c|c|}
\hline \multirow{2}{*}{ G1 } & \multirow{2}{*}{ Graduạ̧ão } & \multirow{2}{*}{$T J$} & \multirow{2}{*}{ STJ } & \multirow{2}{*}{ Esportes na vida } & \multicolumn{8}{|c|}{ Titulos Conquistados } \\
\hline & & & & & RG & IG & SP & $B R$ & AM & MOO & & JAI \\
\hline$S R-66 L C$ & $2^{\circ}$ dan & 17 & $\overline{3}$ & judó, GA e vólei & $\overline{1}$ & 1 & $P$ & $\bar{x}$ & $\bar{x}$ & $\bar{x}$ & $\overline{1}$ & $\bar{P}$ \\
\hline SR -66 MR & $1^{\circ} \mathrm{da}$ & 11 & 5 & judó, jiu-jitsu e fusebol & $i$ & 2 & p & $\mathrm{x}$ & $\mathrm{x}$ & $\mathrm{x}$ & 1 & $\mathrm{P}$ \\
\hline$S R-73 F Z$ & $2^{\circ}$ dan & 10 & 5 & judo & 1 & 1 & $p$ & $x$ & $\mathrm{x}$ & $x$ & 2 & $\mathrm{P}$ \\
\hline$J R-81 T L$ & $1^{\circ} \mathrm{kyu}$ & 8 & 3 & judô, futebol e nataçâo & 1 & 1 & $p$ & $\mathrm{x}$ & $x$ & $\mathrm{x}$ & 2 & $x$ \\
\hline$J R-81 D L$ & $x$ & . & * & $x$ & , & . & $\times$ & . & . & , & . & ' \\
\hline$J R-90 P M$ & $1^{\circ} \mathrm{kgm}$ & 14 & 2 & juodô, tênis e natacâo & 1 & 1 & 3 & $x$ & $x$ & $\mathrm{x}$ & 3 & $\mathrm{P}$ \\
\hline & MEDIA & 12 & $\overline{4}$ & & & & & & & & & \\
\hline & $D P$ & 4 & 1 & & & & & & & & & \\
\hline
\end{tabular}

TABELA 3 - Respostas sobre a vida desportiva dos atletas do $G 2$

\begin{tabular}{|c|c|c|c|c|c|c|c|c|c|c|c|c|}
\hline \multirow{2}{*}{ G2 } & \multirow{2}{*}{ Graduaģăo } & \multirow{2}{*}{$T J$} & \multirow{2}{*}{ STJ } & \multirow{2}{*}{ Esportes na vida } & \multicolumn{8}{|c|}{ Titulos Conquistados } \\
\hline & & & & & RG & & SP & & AM & MOO & & JAI \\
\hline$S R-66 \mathrm{EL}$ & $2^{2}$ dan & 22 & 5 & judêे & 1 & 1 & 1 & 3 & $P$ & $\bar{x}$ & $\frac{1}{1}$ & $\frac{1}{1}$ \\
\hline$S R-81 L F$ & $1^{\circ} \mathrm{dan}$ & 18 & 9 & juob e futebol: & 1 & 1 & 3 & 3 & $x$ & $\mathrm{x}$ & 1 & 2 \\
\hline$S R-90 A K$ & $1^{\circ}$ dan & 28 & 7 & judb e kurash & 1 & 1 & 1 & 1 & 1 & $3+$ & 1 & $i$ \\
\hline SR-90AS & $1^{\circ} \mathrm{dan}$ & 23 & 7 & judá & 1 & 1 & 2 & 3 & $x$ & $\mathrm{x}$ & 1 & 2 \\
\hline \multirow[t]{3}{*}{ JR -66 IS } & $1^{\circ} \mathrm{kys}$ & 7 & 9 & judb, futebol e capoeira & 1 & 2 & 1 & $x$ & $x$ & $x$ & $x$ & $\mathrm{x}$ \\
\hline & MÉDIA & 19 & 7 & & & & & & & & & \\
\hline & $D P$ & 7 & 1 & & & & & & & & & \\
\hline
\end{tabular}

LEGENDAS: TJ: tempo de prática do jucîo (em anos); STJ: sessões de treino de jucóo (vezes por semana); RG: Regional; IG: Inter-Regional; SP: Estadual; BR: Brasileiro; AM: Coutinental; MO Murdial ou Oĺmpico; JR: Jogos Regionais; JAI: Jogos Abertos do Interior; P. participaçào no evento; $\mathrm{X}$ : não participou do evento; $\bullet$ : nào respondeu; 7: Campeonato Mundial Universitário. 
Desempenho dos grupos
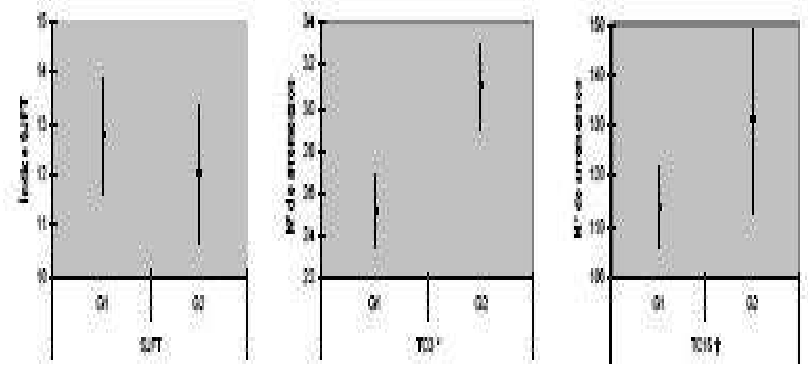

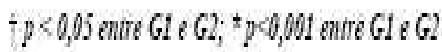

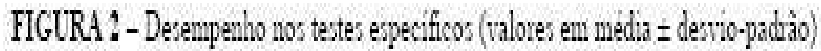

Os resultados encontrados no desempenho nos testes realizados pelos judocas mostraram que no TC3 e no TC15, os grupos apresentaram diferença estatisticamente significante $(p<0,05$ para TC15 e $\mathrm{p}<0,001$ para TC3). Para o resultado do Índice no SJFT, os desempenhos não foram diferentes.

\section{FIDEDIGNIDADE DO TC3}

Os resultados para a reprodutibilidade do TC3 estão dispostos na tabela 4.

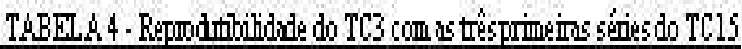

\begin{tabular}{lc}
\hline Reprodutibil:dade & TC $3 \times$ TC15 \\
\hline CCI $0,9 \%$ & $0,00-0,99$ \\
\hline iC $95 \%$ & \\
\hline
\end{tabular}




\section{DISCUSSÕES}

Grupos de judocas

A primeira observação feita a respeito dos grupos é que existe diferença significante quanto a variável Idade $(\mathrm{p}<0,05)$, sendo que G2 apresenta mediana maior que G1. Quanto às variáveis morfológicas, não houve diferença significativa, mostrando que os grupos eram homogêneos. Pela análise das respostas obtidas através do questionário, observa-se maior nível competitivo do G2, pelo fato de que todos os atletas deste grupo possuem títulos estaduais, a maioria possui títulos nacionais e nos Jogos Abertos do Interior, competições consideradas de alto nível dentro do Brasil. Há também de se destacar a classificação em campeonato de nível Mundial de um dos atletas do G2.

\section{DESEMPENHO NOS TESTES}

Através dos resultados encontrados, o desempenho apresentado pelos judocas de ambos os grupos mostrou bastante similaridade e até resultados melhores que os disponíveis em determinados estudos na literatura.

Em estudo de Sterkowicz et al. (1999), os resultados para o volume total foram semelhantes aos encontrados nesse estudo ( $27 \pm 2$ arremessos, média e desvio-padrão, respectivamente, comparados a 26 \pm 3 arremessos do presente trabalho); isso vale também para o Índice $(12,29 \pm 1,48)$, em relação aos judocas neste estudo $(12,29 \pm 1,30)$. Outros trabalhos com judocas de diferentes classes mostraram que o grupo de judocas neste estudo teve melhor desempenho que judocas brasileiros Juvenis, Juniores e Seniores e judocas da Associação Desportiva São Caetano (FRANCHINI, 2001).

Um dos resultados interessantes encontrados na presente pesquisa foi que, ao contrário do encontrado no estudo de Sterkowicz (1996) apud Franchini (2001), os resultados do SJFT mostraram que o desempenho no teste não discriminou os dois grupos de judocas utilizados nesse estudo. No estudo citado, o autor encontrou diferença significante $(\mathrm{p}<0,05)$ entre os resultados de atletas medalhistas e não-medalhistas no Campeonato Polonês de 1994. Para o primeiro grupo, os valores do Índice foram de $11,57 \pm 2,52$, ao passo que no segundo grupo, o resultado foi de 13,28 $\pm 1,34$. Em estudo mais recente, Franchini et al. (2005) constatou que existe diferença estatisticamente significante $(\mathrm{p}<0,05)$ entre grupos de judocas classificados como Elite $(n=23)$ e Não-Elite $(n=53)$, contrapondo os achados do estudo aqui apresentado. Isso abre um precedente para que mais pesquisas sejam realizadas, procurando reforçar ou refutar os achados do presente estudo. 
Os resultados apresentados pelo TC3 mostraram que este teste apresentou diferença altamente significante $(p<0,001)$ entre desempenho dos judocas de G1 e G2, sendo G2 o grupo que teve maior volume de golpes aplicados. Ao observar os valores absolutos, é possível verificar que existe diferença significativa entre o desempenho dos grupos. Os resultados obtidos quanto ao desempenho no TC15 também mostraram diferença estatisticamente significante entre os grupos $(p<0,05)$. Os valores absolutos apresentam diferença entre os grupos, mostrando que tal instrumento de avaliação também diferencia desempenho de judocas.

\section{FIDEDIGNIDADE DO TC3}

Os resultados do TC3 e das três primeiras séries do TC15 quanto à estabilidade, mostram que os judocas não modificaram seu desempenho, mesmo se tratando de testes com volume diferenciado; é possível observar que os atletas mantêm volume de golpes semelhante dentro das três séries de esforço do TC3 e TC15 (valor da mediana 29 e 28 golpes, respectivamente).

Sendo assim, o TC3 parece ser o método de avaliação mais viável no contexto do presente estudo. Além de ser uma tarefa que explora os componentes físico e técnico dos judocas, foi a situação que, através da análise da situação teste-reteste, teve alto grau de reprodutibilidade (CCI 0,97), com intervalos de confiança que foram da ordem de 0,90 a 0,99 . Tais evidências mostram que o TC3 é instrumento fidedigno e pode ser utilizado para medir a condição físico-técnico do judoca.

\section{CONCLUSÕES}

Através das observações conduzidas nesta pesquisa, a respeito dos novos protocolos de avaliação para judocas, concluiu-se que:

1) ao contrário do TC3 e do TC15, o índice proposto pelo SJFT não discriminou judocas, levando em conta o mesmo modelo de classificação dos atletas apresentado por Sterkowicz (1996) apud Franchini (2001);

2) através das evidências, foi comprovada a fidedignidade do TC3 através do método de testereteste (considerado por Thomas e Nelson (2002) como método de equivalência); levando em conta tais fatores, é apresentado como novo protocolo de análise do rendimento físico de judocas;

3) levando em conta o tipo de desgaste provocado para os judocas que são arremessados no TC15, pelo alto volume de golpes aplicados, o teste deve ter outra estrutura, procurando desenvolver formas alternativas de avaliação a partir da proposta inicial; 
4) novos estudos com os dois métodos de avaliação propostos devem ser conduzidos, para atestar a validade frente a modelos de periodização aplicados em judocas, procurando, assim, constatar se os testes são sensíveis a mudanças funcionais, provocados por adaptações induzidas através do treinamento sistematizado de judô.

\section{REFERÊNCIAS}

BAR-OR, O. The Wingate anaerobic test: an update on methodology, reliability and validity. Sports Medicine, v. 4, p. 381-394, 1987.

BOMPA, T. O. Periodization training for sports. Champaign: EUA, Human Kinetics, 1999.

BORKOWSKI, L.; FAFF, J.; STARCZEWSKA-CZAPOWSCA, J. Evaluation of the aerobic and anaerobic fitness in judoists from the Polish national team. Biology of Sport, v. 18, n. 2, p. 107-118, 2001 .

CALLISTER, R. et al. Physiological and performance responses to overtraining in elite judo athletes. Medicine and Science in Sports and Exercise, v. 22, n. 6, p. 816-824, 1990.

. et al. Physiological characteristics of elite judo athletes. International Journal of Sports Medicine, v. 12, p. 196-203, 1991.

CAVAZANI, R. N. Lactato antes e após sucessivos combates de judô. 1991. Monografia (Bacharelado em Educação Física), Faculdade de Educação Física, Instituto de Biociências, Universidade Estadual Paulista, 1991.

DEGOUTTE, F.; JOUANEL, P.; FILAIRE, E. Energy demands during a judo match and recovery. British Journal of Sports Medicine, v. 37, p. 245-249, 2003.

DE MEERSMAN, R. E.; RUHLING, R. O. Effects of judo instruction on cardiorespiratory parameters. Journal of Sports Medicine, v. 17, p. 169-172, 1977.

FRANCHINI, E. et al. Características fisiológicas em testes laboratoriais e resposta da concentração de lactato sangüíneo em 3 lutas em judocas das classes juvenil, junior e sênior. Revista Paulista de Educação Física, São Paulo, v. 12, n. 1, p. 5-16, 1998.

. Judô: desempenho competitivo. São Paulo, Manole, 2001.

.Tipo de recuperação após a luta, diminuição do lactato e desempenho posterior: implicações para o judô. 2001a Tese (Doutorado). Escola de Educação Física e Esporte, Universidade de São Paulo, São Paulo, 2001a.

E. et al. S. Physical fitness and anthropometrical differences between elite and non-elite judo players. Biology of Sport, v. 22, n. 4, p. 315-328, 2005. 
KUZNETSOV, V. V. Metodologia del entrenamiento de la fuerza para deportistas de alto nivel. Buenos Aires: Stadium, 1986.

LITTLE, N. G. Physical performance attributes of Junior and senior women, juvenile, junior and senior men judokas. Journal of Sports Medicine and Physical Fitness, v. 31, p. 510-520, 1991.

MATVEEV, L. P. Fundamentos del entrenamiento deportivo. Madrid: Maluar, 1980.

SIKORSKI, W.; MICKIEWICZ, G.; MAJLE, B.; LAKSA, C. Structure of the contest and work capacity of the judoist. In: Proceedings of the International Congress on Judo "Contemporary Problems of Training and Judo Contest", Spala, Poland, p. 58-65, 1987

SILVA, W. N. C. Judô: Estudo das ações motoras específicas da competição: Estratégias metodológicas para o treinamento. 2002. Dissertação (Mestrado) - Faculdade de Educação Física, Universidade Estadual de Campinas, Campinas, 2002.

STERKOWICZ, S.; ZUCHOWICZ, A.; KUBICA, R. Levels of anaerobic and aerobic capacity indices and results for the Special Judo Fitness Test in judo competitors. In: Annals of the Coach's Professional Activities - Managing the Training Process in Combat Sports - Express Scientific Conference, Cracow, Poland, p. 6-26, 1999.

STERKOWICZ, S.; FRANCHINI, E. Techniques used by judoists during the World and Olympic tournaments 1995 - 1999. Human Movement, v. 2, n. 2, p. 24-33, 2000.

TAYLOR, A. W.; BRASSARD, L. A physiological profile of the Canadian Judo Team. Journal of Sports Medicines. v. 21, n. 1, p. 160-164, 1981.

ThOMAS, S. G.; COX, M. H.; LEGAL, Y. M.; VERDE, T. J.; SMITH, H. K. Physiological profiles of Canadian National Judo Team. Canadian Journal of Sports Sciences, v. 14, n. 3, p. 142-147, 1989.

THOMAS, J. R.; NELSON, J. K. Métodos de pesquisa em atividade física. 3. ed. Porto Alegre: Artmed, 2002.

TUMILTY, D. M.; HAHN, A. G.; TELFORD, R. D. A Physiological profile of well- trained male judo players. In: Watkins, J.; Reilly, T.; Burwitz, L. (Ed.). Proceedings of the VIII Commonwealth and International Conference on Sport, Physical Education, Dance, Recreation and Health. London, E \& F. N. Spon., p. 3-10, 1986.

VERKOSHANSKY, Y. V. Entrenamiento deportivo: planificacion e programacion. Barcelona: Martinez Roca, 1990.

ZATSIORSKY, V. M. Ciência e prática do treinamento de força. São Paulo: Phorte, 1999.

Conexões, revista da Faculdade de Educação Física da UNICAMP, Campinas, v. 6, ed. especial, p. 11- 21, jul. 2008. 


\section{Rodrigo Ribeiro Rosa}

Faculdade de Educação Física/UNICAMP

\section{Paulo Roberto de Oliveira}

Faculdade de Educação Física/UNICAMP

\section{Referência do artigo:}

\section{ABNT}

ROSA, R. R.; OLIVEIRA, P. R. Novas propostas de avaliação da resistência especial do judoca. Conexões, v. 6, ed. especial, p. 11-21, 2008.

\section{APA}

Rosa, R. R., Oliveira, P. R. (2008). Novas propostas de avaliação da resistência especial do judoca. Conexões, v. 6, (ed. especial), 11-21, 2008.

\section{VANCOUVER}

Rosa RR, Oliveira PR. Novas propostas de avaliação da resistência especial do judoca. Conexões, 2008; v. 6, (ed. especial): 11-21. 